\title{
The role of PLUNC proteins in the etiopathogeny of chronic rhinosinusitis
}

\author{
Claudiu Manea ${ }^{1,2}$, Codrut Sarafoleanu ${ }^{1,2}$, Cristina losif', Elena Patrascu ${ }^{1,2}$ \\ ${ }^{1}$ ENT\&HNS Department, "Sfanta Maria” Hospital, Bucharest, Romania \\ 2"Carol Davila" University of Medicine and Pharmacy, Bucharest, Romania
}

\section{ABSTRACT}

BACKGROUND. Innate immunity represents the first way of protection against different pathogenic agents. Recently, it has been a permanent interest regarding an innate immune molecule, that is known as palate, lung, nasal epithelial clone (PLUNC). PLUNC is a specific result found in the airways, of approximately $25 \mathrm{kDa}$, whose encoding is realized by adjacent genes located within a region of $300 \mathrm{~kb}$ in chromosome 20; these proteins must be detected predominantly in the superior respiratory tract.

MATERIAL AND METHODS. We performed a prospective clinical study on 34 patients with chronic rhinosinusitis and 10 controls, in order to investigate the expression of this protein in nasal tissue of patients affected by chronic rhinosinusitis. We tested the correlation between the existence of this disease and PLUNC proteins positivity.

RESULTS. $100 \%$ of controls have a+++ rated PLUNC proteins positivity, while cases have a lower percentage of positivity. There were no differences statistically significant between patients with CRSwNP and those with CRSsNP.

CONCLUSION. As a response to different irritating agents (bacteria, viruses, chemical factors), nasal mucosa will react by producing PLUNC proteins. PLUNC proteins have a defensive role in the upper airways mucosa.

KEYWORDS: PLUNC proteins, etiopathogeny, chronic rhinosinusitis, superior respiratory tract

\section{INTRODUCTION}

Rhinosinusitis is an important chronic disease, being characterized by a persistent inflammation of the nasal and of the paranasal sinuses mucosa. Chronic rhinosinusitis (CRS) with or without nasal polyposis is a multifactorial disease with various agents (mechanical, viral, bacterial, fungal infection, immunological disorders, hyperreactivity, pollution) acting on the nasal mucosa, that are able to initiate epithelial damage and maintain chronic inflammation.

Worldwide researchers are permanently trying to find new molecules involved in the etiopathogeny of chronic rhinosinusitis, in order to provide and indicate the optimal treatment, with maximum efficacy.

In multicellular organisms, innate immunity is the first-line method of protection against external agents. The innate immune system is formed of a group of polypeptides and proteins that collaborate, in order to respond to microbes and/or chemical agents. The upper airways, including oral and nasal cavities, are the primary way of entrance of pathogens into the body and this is why precocious recognition of antigens in these areas is essential for a good host defence. These molecules are often produced in these regions, as well as in the major and minor salivary glands of the oral cavity, gastric mucosa and in the skin ${ }^{1}$.

After the action of external microorganisms, many cell subtypes, including neutrophils and macrophages, react by releasing inflammatory molecules or by becoming activated, with the purpose of inhibiting bacterial invasion. When bacterial antigens are found in a relatively high quantity, responses may become strong enough to determine sepsis, followed by different complications and high mortality.

In majority of the studies, greatest attention was paid to bacterial constituents, represented by lipopolysaccharides (LPS), which are recognized by specific 
receptor complexes located on the surfaces of activated cells (neutrophils, macrophages) only if they are bound to a secreted protein that acts as a presentation agent for the respective receptor ${ }^{1,2}$.

In human subjects, there are two proteins with an important role in defence against bacteria, by mediating the signals from LPS - bactericidal/permeabilityincreasing protein (BPI) and LPS-binding protein (LBP). These two proteins seem to be related from a structural point of view and they may have antagonistic ways of action ${ }^{1,3}$. LBP binds LPS and determines cellular host reactions to LPS, while BPI binds LPS, but it has a role of reducing cellular responses ${ }^{4}$. By this mechanism, a balance is maintained in the response to bacterial action. Apart from neutralizing LPS, BPI also has a different way of destroying bacterial antigens directly ${ }^{3}$. Two other related proteins, phospholipid transfer protein (PLTP) and cholesteryl ester transfer protein (CETP) have also been involved in performing LPS responses.

Despite the fact that LBP is a circulating agent, it has been suggested that a greater local source of LPSsensing molecules located in the respiratory airways would facilitate a more immediate response to inhaled pathogens.

Recent studies have tried to identify new molecules that play a role in the host defence against pathogens, acting upon the innate immune system.

A novel protein, whose function in inflammatory pathologies of the upper respiratory tract has been recently investigated, called PLUNC (palate, lung and nasal epithelium clone), is secreted by epithelia of conducting airways, related to the LT/LBP family. This family plays an important role in detecting and responding to Gram-negative bacteria. It leads to the opinion that PLUNC may play a defensive role in the human respiratory tract. It may also act as a sensor of bacteria in the superior airways, as well as in the oral cavity, because it has been proven that PLUNC genes are expressed mostly in these sites.

PLUNC mRNA has been identified in upper airways mucosa, while his expression is absent in the inferior airways and lung periphery. Genes that encode PLUNC proteins are located in a region of $300 \mathrm{~kb}$ belonging to chromosome $20^{2}$, suggesting the fact that the transcription of these proteins could be under control of common genomic elements.

Data available in literature prove that PLUNC proteins are found in overlapping sites of the pulmonary, nasopharyngeal and oral epithelium, including saliva and nasal lavage fluid, areas where bactericidal or permeability-increasing proteins (also components of the innate immune system protein), are not present. PLUNC has a molecular weight of approximately $25 \mathrm{kDa}$.

When a subject is exposed to airway irritants for a long period of time, a decrease of PLUNC levels in the upper airways is produced, probably because it has a toxic effect upon secretory cells. From all the proteins that are present only in human nasal lavage, PLUNC proteins are the only ones who were adsorbed on a lipopolysaccharide-coated surface, which is essential for providing an optimal protection against Gramnegative bacteria ${ }^{3,4}$

Members belonging to PLUNC family have been categorized (and named) according to their sizes - short (SPLUNC) and long (LPLUNC) proteins. Short proteins are represented by SPLUNC-1 (Short PLUNC-1; 256 amino acids), SPLUNC-2 (Short PLUNC- 2; 249 amino acids), and SPLUNC-3 (Short PLUNC-3; 253 amino acids). Long proteins are represented by LPLUNC-1 (Long PLUNC-1; 484 amino acids), LPLUNC-2 (Long PLUNC-2; 458 amino acids), LPLUNC-3 (Long PLUNC3; 463 amino acids), and LPLUNC-4 (Long PLUNC-4; $>469$ amino acids). Human PLUNC family evolve very quickly, which sustains the idea that these proteins play an important role in host defence.

The short proteins present homology only to the N-terminal domain of bactericidal/permeability-increasing protein, whilst long proteins are similar to both the $\mathrm{N}$ - and C-terminal domains of bactericidal/ permeability-increasing protein, as well as LBP ${ }^{5}$.

Primary protein sequence is pretty similar to an equine protein, called latherin. Latherin, who was originally isolated from horse sweat, actually is proven to be present in saliva, where it has surfactant effects on air or on liquid interfaces. Equine latherin belongs to PLUNC family, which gives it a certain degree of hydrophobicity. Hydrophobic residues have a similar proportion of the total amino acid content in latherin and PLUNC (44.2\% in latherin, $44.7 \%$ in PLUNC) ${ }^{6}$.

PLUNC proteins are also known as SPLUNC-1 (Short PLUNC-1), LUNX (lung-specific X protein), NASG (nasopharyngeal carcinoma-related protein) or SPURT (secretory protein in upper respiratory tracts).

During the past years, people have had a great interest in finding the role of PLUNC protein in airway inflammation. The main idea began with its important hydrophobicity and its expression pattern, so it has been thought that PLUNC is an airway surfactant that also accomplishes an antibiofilm activity, by blocking biofilm formation, as a response to the most frequent aeropathogens; furthermore, it can be upregulated after bulbectomy, in order to prevent appearance of an infection after trauma ${ }^{7,8}$.

The antimicrobial effect of PLUNC and its surfactant activity of reducing the surface tension of the airways mucosa may represent a novel form of innate immunity that limits bacterial colonization of the airways; a malfunctioning of the innate immune molecules could act as a stimulating factor in chronic rhinosinusitis pathogenesis, creating an optimal condition for further microbial afflux ${ }^{9}$. 
PLUNC positivity was markedly reduced in the mucosal epithelia and in the submucosal glands, among the patients with multibacterial infections, especially those who were Staphylococcus aureus- and Pseudomonas aeruginosa-mediated. These data suggest that patients with chronic rhinosinusitis and with reduced PLUNC expression might have an immune defect in fighting against bacterial infection; subsequently, low PLUNC expression can lead to recurrent Staphylococcus aureus and Pseudomonas aeruginosa infections. So, low levels of PLUNC expression in airways tissues could be considered as a mucosal inflammatory measurement, to evaluate the inflammation of sinusal mucosa, which might appear because of a Staphylococcus aureus and Pseudomonas aeruginosa colonization.

There are various mechanisms that link PLUNC levels and expression with the inflammation in patients with microbial infections. There are studies that show that PLUNC protein is secreted by neutrophils, after bacterial colonization ${ }^{10}$. PLUNC protein participates to antimicrobial host defence, via both bactericidal and non-bactericidal ways of action, also by reducing the production of IL- 8 , IL-6, IL- $1 b^{11,12}$.

The discovery and study of PLUNC protein might represent a new predictive outcome factor for patients with chronic rhinosinusitis, after bacterial colonization. In patients with chronic rhinosinusitis, in which we found low levels of PLUNC positivity, we must apply an antibiotic treatment, based on the results of antibiograms and we must use efficient topic treatment (nasal and sinusal irrigation) to minimize the possibility of chronicization of this pathology.

Although the regulation of PLUNC protein is not completely discovered, it has been noticed that levels of PLUNC gene and protein expression are not significantly modified after applying IL-1b and TNF-a to normal human nasal epithelial cells. Moreover, PLUNC proteins expression is not modified by different inflammatory degrees of the nasal and paranasal sinuses mucosa. These results suggest that different inflammatory molecules may not influence PLUNC expression, but raise an idea that there is a permanent secretion of PLUNC protein ${ }^{13}$.

We can suggest that decrease in the expression of PLUNC proteins is able to contribute to chronic rhinosinusitis pathogenesis, not only as an effect of reducing antimicrobial effects, but also by altering its physical and chemical effects.

Recent theories regarding the etiopathogeny of chronic rhinosinusitis not only involve malfunctioning of the innate immune system factors, barrier defects and the role for superantigens, but also different mucociliary dysfunctions. PLUNC proteins have a role in inhibiting the epithelial sodium channels (ENaC) activation, with effects upon the mucociliary clearance system $^{14}$. The malfunctioning of ENaC may cause a dysregulation of the mucociliary clearance and this can be considered another mechanism by which pathogens determine and sustain chronic inflammation.

\section{MATERIAL AND METHODS}

We performed a prospective clinical study, in order to determine the level of PLUNC protein in nasal samples prelevated from patients diagnosed with chronic rhinosinusitis.

For the diagnosis of chronic rhinosinusitis, we used criteria proposed by EPOS guide: the presence of two or more symptoms, a major one represented by nasal blockage/ obstruction/congestion or by nasal discharge (anterior/posterior nasal drip) ${ }^{15}$. Minor symptoms could be facial pain or pressure, with or without reduction or loss of smell.

Nasal endoscopy assessed the evidence of polyps; mucopurulent secretions mostly coming from the middle meatus; oedema/mucosal obstruction, especially in the middle meatus; transformation of the mucosa of the ostiomeatal complex and/or sinuses noticed by CT scan. Nasal polyps degree was classified according to Lund-MacKay grading system.

We included in our study 34 patients with chronic rhinosinusitis without (14 patients) and with nasal polyps (20 patients), who underwent surgery in "Sfanta Maria" Hospital, Bucharest. For comparative purposes, we prelevated nasal biopsies from 10 healthy patients, who had no symptoms of chronic rhinosinusitis and who were not diagnosed with any other hypersensitivities.

Patients included in our study group were diagnosed with CRS based on clinical and paraclinical investigation (computer tomography, nasal endoscopy, histopathological disease confirmation). Study exclusion criteria were: hematologic diseases, pregnancy.

We evaluated patients, considering the age group, clinical history, severity of the symptoms and smoking habits. Patients were also questioned about presence or absence of allergy, in terms of NSAIDs intolerance, allergic rhinitis, asthma or other allergic manifestations (mostly, antibiotic allergy). Skin prick tests including aeroallergens were performed, to evaluate the presence of allergy, while asthma was diagnosed by a pneumologist based on the evaluation of airway responsiveness. All smoker patients had moderate smoking habits (from 10 to 20 cigarettes per day).

Tissues were taken from the ethmoid sinus of cases, while normal mucosal tissues were obtained from the turbinate mucosa for the 10 controls. We focused on the most highly expressed PLUNC family member, SPLUNC-1, to elucidate its expression in chronic rhinosinusitis. All the samples were paraffin-embedded. To ascertain the intensity of PLUNG protein expres- 
$\operatorname{sion}^{16}$, we deparaffinized, dehydrated with graded alcohol series, and rehydrated them. All samples were then coloured with streptavidin-peroxidase method. After that, they were blocked for 30 minutes with $0.3 \%$ $\mathrm{H} 2 \mathrm{O} 2$ in methanol and incubated with serum for 1 hour. The sections were then incubated for 30 minutes with $0.1 \mathrm{~mL}$ of goat polyclonal antibody against PLUNC protein ( $1: 200$ dilution; R\&D Systems, Minneapolis), followed by a Rabbit Anti-Goat IgG HRP Affinity Purified PAb (R\&D Systems, Minneapolis) for another 30 minutes.

The sections were then colorized with diaminobenzidine/ hydrogen peroxide and counterstained with hematoxylin. Finally, the sections were examined under a microscope (40-100x).

We classified the intensity of PLUNC positivity, according to the distribution percentage, as following: $+++(>50 \%$ positive cells $),++(26$ to $50 \%$ positive cells $)$, $+(\leq 25 \%$ positive cells) or - (no positive cells).

Statistical analysis was performed by using the statistical package SPSS version 15.0. A Student t-test or a Mann-Whitney test was used for comparison of two different groups and an ANOVA test or Kruskall-Wallis test for more than two groups. Kendall's tau-b correlation was used to assess correlation. We considered a p-value less than 0.05 statistically significant. Data obtained are presented as mean \pm SD and median.

We evaluated the correlation between patients and control subjects for frequencies of PLUNC proteins positivity, using the $\chi 2$ test with statistical significance level of $95 \%$ (p-value $<0.05)$.

\section{RESULTS}

Among the patients included in our study group, 24 were male $(70.58 \%)$ and 10 were female $(29.41 \%)$. The ages ranged between 23 and 68 years, with an av-

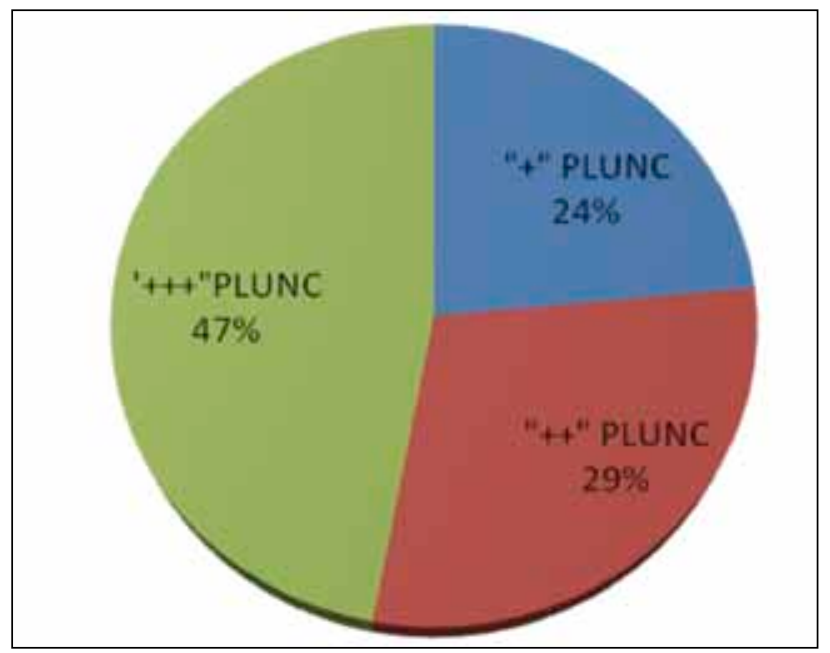

Figure 1 PLUNC protein positivity in patients with CRS erage age of 47.6 years. Control subjects were 6 male and 4 female, aged between 19 and 56 years.

Regarding the potential risk factors involved in the etiopathogeny, as well as in the evolution and severity of chronic rhinosinusitis, only 4 patients $(11.76 \%)$ were smokers.

In order to identify the importance of allergic conditions, patients were evaluated for the presence of different atopies. Twelve patients (10 male, 2 female) $-35.29 \%$ were overall allergy sufferers. 9 patients $(5$ male, 4 female) had asthma, and 6 patients ( 3 male, 3 female) were known with NSAIDs intolerance.

In our study, we investigated predominantly the most frequent expressed molecules belonging to PLUNC family in paranasal sinuses and nasal tissues, SPLUNC-1. We found low levels in the nasal mucosa samples in case of the patients diagnosed with chronic rhinosinusitis, probably because of a decreased number of secretory glands.

In our study, we did not obtained any statistically significant correlation between PLUNC proteins expression and the presence of risk factors, in terms of different allergy manifestations, asthma, neither in case of smoking habits. Other studies found in the literature suggest there may be a correlation between PLUNC positivity, allergy and smoking habits in patients with various inflammatory diseases.

The results of the present study show that we have a high positivity $(+++)$ in the control group $(100 \%$ of the cases), while the patients with chronic rhinosinusitis have a significant lower PLUNC proteins positivity (Figure 1).

Only $47.06 \%$ of the case patients have a +++ rated positivity (Figure 2), whilst the rest of the patients have $\mathrm{a}++(29.41 \%)$ or $+(23.52 \%)$ rated positivity (Figure 3 , Figure 4). We did not observe a negative value of PLUNC proteins, neither in the study group, nor in control patients.

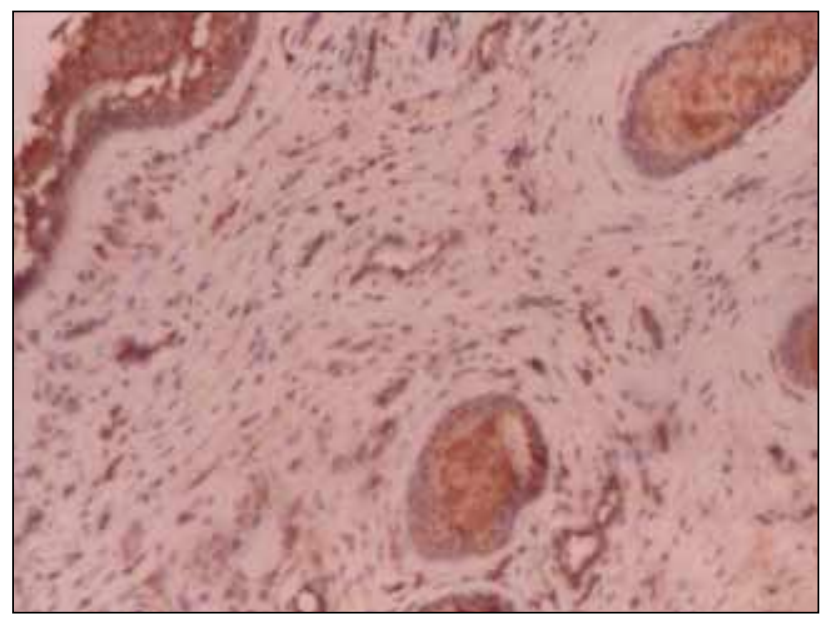

Figure 2 Nasal mucosa PLUNC protein positivity +++ in respiratory epithelium, glandular, lymphoid elements from stroma, IHC, 10x 


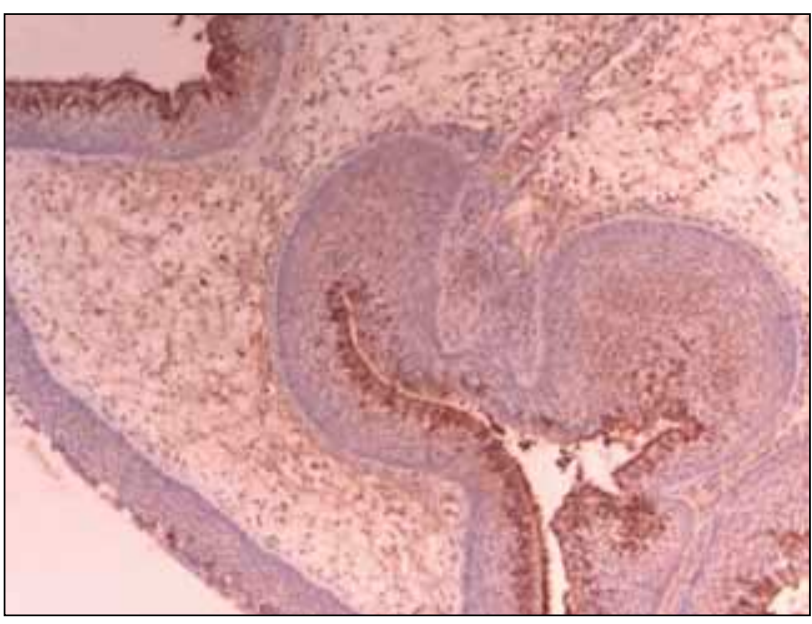

Figure 3 Nasal mucosa - PLUNC focal positivity (+) in epithelial cells, stroma, oedema, IHC $4 \mathrm{x}$

We examined the correlation between the presence of this disease and the PLUNC proteins positivity using $\chi 2$ statistical test to analyze the results of the study. We observed that the differences in PLUNC proteins positivity between patients with CRS and people from the control group are statistically significant ( $\chi 2$ test, pvalue $=0.002)($ Table 1$)$.

When comparing the patients with CRSwNP and CRSsNP, we did not obtain any statistically significant difference between the two entities $(\mathrm{P}$ value $=0.280$ ).

\section{DISCUSSIONS}

The PLUNC family, a newly discovered gene, expressed in the upper airways, plays an important role in the mechanisms of host defence against bacteria, being a part of the innate immune mechanisms in the oro-nasal mucosa, as well as in the pulmonary epithelium, sites exposed to a high amount of bacterial antigens, as it was postulated in the latest scientific studies.

\section{Table 1}

PLUNC proteins positivity in patients and controls

\begin{tabular}{|c|c|c|c|c|c|}
\hline & & & \multicolumn{3}{|l|}{ Lot } \\
\hline & & & CRS & control & Total \\
\hline \multirow{8}{*}{$\begin{array}{c}\text { PLUNC } \\
\text { positivity }\end{array}$} & + & Count & 8 & 0 & 8 \\
\hline & & $\%$ & $23.5 \%$ & $.0 \%$ & $18.2 \%$ \\
\hline & ++ & Count & 10 & 0 & 10 \\
\hline & & $\%$ & $29.4 \%$ & $.0 \%$ & $22.7 \%$ \\
\hline & +++ & Count & 16 & 10 & 26 \\
\hline & & $\%$ & $47.1 \%$ & $100.0 \%$ & $59.1 \%$ \\
\hline & Total & Count & 34 & 10 & 44 \\
\hline & & $\%$ & $100.0 \%$ & $100.0 \%$ & $100.0 \%$ \\
\hline
\end{tabular}

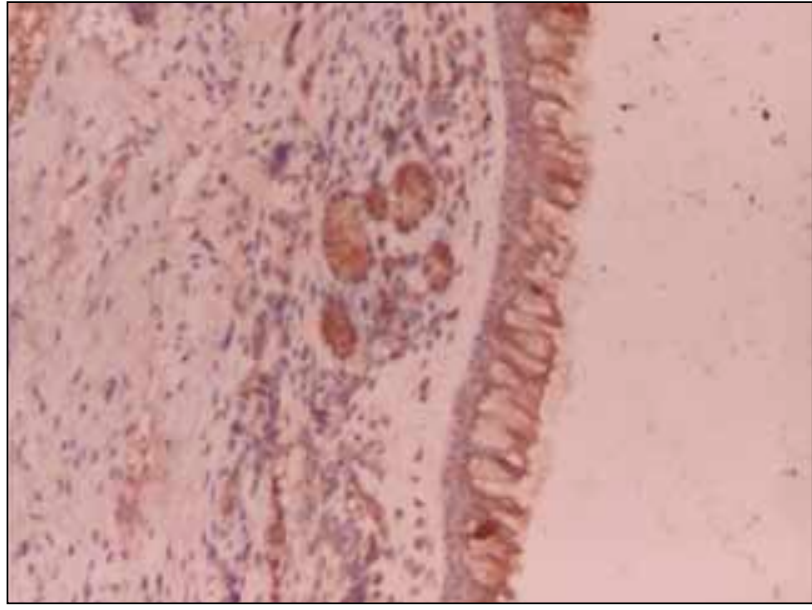

Figure 4 Nasal mucosa - PLUNC positivity in respiratory epithelium, in mucosal glands, in inflammatory elements, IHC 10x

In the literature, there are some studies that indicate that low levels of PLUNC protein were found in human nasal lavage fluid from smoking persons and people who work with reactive epoxy substances, proving that exposure on a long term to airway irritant factors may impair the expression of PLUNC in the superior respiratory tract. In human nasal lavage fluid, there have been identified two forms of the PLUNC protein using two-dimensional gel electrophoresis.

It has been also studied the existence of lipopolysaccharide-binding proteins in human nasal lavage fluid. There were found five proteins that were able to adsorb to a LPS-coated surface; two of the proteins found correlate to the two PLUNC forms (short and long). Human saliva was also investigated for the presence of LBP and it has been proven that it contains a certain subset of LPS-binding proteins with similar localizations. Actual results sustain that PLUNC could be a new marker of upper airways inflammation and could be a part of the innate immune molecules.

In other studies, researchers have found that PLUNC proteins are able to present high surfactant activity in different degrees. Also, experiments using epithelial cells of upper airway tract and primary cultures show that native PLUNC exhibit a powerful activity of reducing the surface tension in aqueous solutions of the airway epithelial secretions. Surface tensions obtained after adding PLUNC to aqueous solutions are quite alike to surface tension values in tracheo-bronchial secretions from humans and animals.

Beside all these properties, PLUNC proteins also have an ability to inhibit biofilm formation in an in vitro-model, in a physiologically relevant concentration, after infection with Pseudomonas aeruginosa, without really having a bactericide function. In order to survive, an early recognition of bacterial antigens is essential. Lipopolysaccharide (LPS), parts of bacterial cell walls, like those present on the surface of Gram- 
negative bacteria, lipoteichoic acids in Gram-positive bacteria and lipoarabinomannans in mycobacteria have important clinical effects in antigen recognition. Because surfactants from the surface of microbes are able to disperse matrix-encased bacterial clusters (biofilms) ${ }^{1,17}$, it was suggested that PLUNC may also possess anti-biofilm properties. The capacity of inhibiting biofilm formation by airway pathogens may be explained by the dispersant properties of PLUNC protein family.

Correlated data from the literature show that PLUNC is a multifunctional protein, with a great new role in airway defences at the interfaces between air and liquids.

\section{CONCLUSIONS}

In our study of 44 patients, the results of our statistical analysis show that the degree of PLUNC protein positivity is, statistically significantly, correlated to the presence of chronic rhinosinusitis with or without nasal polyps, with no difference between the two entities. That suggests that, after injury (infectious pathogens, chemical factors), the nasal mucosal epithelium will react and will generate antiinflammatory agents, including PLUNC proteins, as a response to the irritant agents ${ }^{13,18}$. Afterwards, the secretion of these antiinflammatory agents is upregulated, to function as a defensive mechanism.

In the cases when defects appear in the immune defence or it happens a malfunctioning of the respiratory mucosa, it may appear a vicious circle of the tissue inflammation, and pathologies characterized by infection and/or inflammation may appear. After the mucosa is injured, it appears a decrease in the number of glands in affected tissues, with a subsequent decrease of the secretion of host defence molecules that determines a decreased capability of clearing the pathogenic factors involved. These defects of defence in the immune system may lead to a persistent inflammatory response that underlies the pathogenic mechanism encountered in chronic rhinosinusitis.

The results we obtained in our study stand as a basis for further studies of this new family of proteins and represent one step ahead in finding new molecules involved in the pathogeny of chronic inflammation of the upper airway tract, in order to better understand the disease and to initiate the optimal treatment. The main idea of the recent studies still remains the possibility that PLUNC may have a great role in the innate immune response of the superior airway tract. It is possible in the future that PLUNC could be seen as a marker of airway affection and that decreased levels of PLUNC could promote inflammation of the nasal mucosa.

\section{REFERENCES}

1. Bingle C.D., Craven C.J. - PLUNC: a novel family of candidate host defence proteins expressed in the upper airways and nasopharynx. Hum Mol Genet., 2002;11(8):937-943.

2. Bingle C.D, Gorr S.U. - Host defence in oral and airway epithelia: chromosome 20 contributes a new protein family. Int J Biochem Cell Biol., 2004;36(11):2144-2152.

3. Ghafouri B., Kihlstrom E., Stahlbom B., Tagesson C., Lindahl M. - PLUNC (palate, lung and nasal epithelial clone) proteins in human nasal lavage fluid. Biochem Soc Trans., 2003;31 (Pt 4):810-814.

4. Passali D., Sarafoleanu C., Manea C., Loglisci M., Passali F.M., Cambi J., Iosif C., Panaitescu E., Bellussi L.M. - PLUNC Proteins Positivity in Patients with Chronic Rhinosinusitis: A Case-Control Study. The Scientific World Journal, Vol. 2014, Article ID 853583, 5 pages. http://dx.doi.org/10.1155/2014/853583

5. Bingle C.D., Seal R.L., Craven C.J. - Systematic nomenclature for the PLUNC/PSP/BSP30/SMGB proteins as a subfamily of the BPI fold-containing superfamily. Biochem Soc Trans., 2011;39(4):977-983. doi: 10.1042//BST0390977.

6. Kennedy M.W. - Latherin and other biocompatible surfactant proteins. Biochem Soc Trans., 2011;39(4):1017-1022. doi: 10.1042/BST0.391017.

7. Gakhar L., Bartlett J.A., Penterman J., et al. - PLUNC is a novel airway surfactant protein with anti-biofilm activity. PLoS One, 2010;5(2):e9098. doi: 10.1371/journal.pone.0009098.

8. Sung Y.K., Moon C., Yoo J., Pearse D., Pevsner J., Ronnett G.V. - Plunc, a member of the secretory gland protein family, is up-regulated in nasal respiratory epithelium after olfactory bulbectomy. J Biol Chem., 2002;277(15):12762-12769. Epub 2002 Jan 30.

9. Bartlett J., Gakhar L., Penterman J., et al. - PLUNC: a multifunctional surfactant of the airways. Biochem Soc Trans., 2011;39(4):1012-1016. doi: 10.1042/BST0391012.

10. Bartlett J.A., Hicks B.J., Schlomann J.M., Ramachandran S., Nauseef W.M., McCray P.B. Jr. - PLUNC is a secreted product of neutrophil granules. J Leukoc Biol., 2008;83(5):1201.1206. doi: 10.1189/jlb.0507302. Epub 2008 Feb 1.

11. Lukinskiene L., Liu Y., Reynolds S.D., et al. - Antimicrobial activity of PLUNC protects against Pseudomonas aeruginosa infection. J Immunol., 2011;187(1):382-390. doi: 10.4049/jimmunol.1001769. Epub 2011 Jun 1.

12. Chu H.W., Thaikoottathil J., Rino J.G., et al. - Function and regulation of SPLUNC1 protein in mycoplasma infection and allergic inflammation. J Immunol., 2007;179(6):3995-4002.

13. Kim C., Kim K., Kim H.J., Kim J.K., Lee J., Yoon J. - Expression and regulation of PLUNC in human nasal epithelium. Acta Otolaryngol., 2006;126(10):1073-1078.

14. Garcia-Caballero A., Rasmussen J.E., Gaillard E., et al. - SPLUNC1 regulates airway surface liquid volume by protecting ENaC from proteolytic cleavage. Proc Natl Acad Sci U S A., 2009;106(27):11412-11417. doi: 10.1073/pnas.0903609106. Epub 2009 Jun 18.

15. Fokkens W.J., Lund V.J., Mullol J., et al. - EPOS 2012: European position paper on rhinosinusitis and nasal polyps 2012. A summary for otorhinolaryngologists. Rhinology, 2012;50(1):1-12. doi: 10.4193/Rhino50E2.

16. Wu M., Sun H., Nan Q. - Expression and clinical significance of PLUNC protein in nasal polyp and chronic sinusitis tissue. Ear Nose Throat J., 2012;91(7):282-285.

17. Irander K., Borres M.P., Ghafouri B. - The effects of physical exercise and smoking habits on the expression of SPLUNC1 in nasal lavage fluids from allergic rhinitis subjects. Int J Pediatr Otorhinolaryngol., 2014;78(4):618622. doi: 10.1016/j.ijporl.2014.01.014. Epub 2014 Jan 23.

18. Bingle C.D., Wilson K., Lunn H., et al. - Human LPLUNC1 is a secreted product of goblet cells and minor glands of the respiratory and upper aerodigestive tracts. Histochem Cell Biol., 2010;133(5):505-515. doi: 10.1007/s00418-010-0683-0. Epub 2010 Mar 18. 